Table 4-1. Parameters for the comparison problem of imbibing into a single-matrix block

\begin{tabular}{|c|c|c|}
\hline Parameter & Value & Unit \\
\hline Effective matrix porosity & $\phi_{M}=0.30$ & \\
\hline Absolute matrix permeability & $\begin{array}{c}\mathrm{k}_{\mathrm{M}}=1.0 \times 10^{-} \\
15\end{array}$ & $\mathrm{~m}^{2}$ \\
\hline Water density & $\rho_{w}=1,000$ & $\mathrm{~kg} / \mathrm{m}^{3}$ \\
\hline Water viscosity & $\mu_{w}=1.0 \times 10^{-3}$ & Paøs \\
\hline Residual matrix saturation & $\mathrm{S}_{\mathrm{wr}, \mathrm{M}}=0.2$ & \\
\hline Initial matrix saturation & $\mathrm{S}_{\mathrm{Mi}}=0.2$ & \\
\hline Saturation on matrix surface & $S_{b}=0.2$ and 0.8 & \\
\hline $\begin{array}{c}\text { Coefficient of matrix relative } \\
\text { permeability }\end{array}$ & $C_{k}=1.0$ & \\
\hline $\begin{array}{c}\text { Exponent of matrix relative } \\
\text { permeability }\end{array}$ & $\alpha=2.0$ & \\
\hline $\begin{array}{c}\text { Coefficient of matrix capillary } \\
\text { pressure }\end{array}$ & $\mathrm{C}_{\mathrm{p}, \mathrm{M}}=1.0 \times 10^{4}$ & $\mathrm{~Pa}$ \\
\hline $\begin{array}{c}\text { Exponent of matrix capillary } \\
\text { pressure }\end{array}$ & $\beta=1.0$ & \\
\hline Initial fracture saturation & $\mathrm{S}_{\mathrm{Fi}}=0.012$ & \\
\hline Residual fracturex saturation & $S_{\mathrm{Fr}}=0.01$ & \\
\hline $\begin{array}{l}\text { Fracture van Genuchten } \\
\text { constant } m\end{array}$ & $m_{\mathrm{vG}}=0.611$ & \\
\hline Fracture van Genuchten $\alpha$ & $\alpha_{v G}=1.0 \times 10^{-3}$ & $\mathrm{~Pa}^{-1}$ \\
\hline
\end{tabular}


Table 4-2. Parameters for the comparison problem with radial unsaturated flow in fractured formation

\begin{tabular}{|l|l|l|l|}
\hline Parameter & Matrix & Fracture & Unit \\
\hline Matrix dimension & $\mathrm{B}=1$ & & $\mathrm{~m}$ \\
\hline Porosity & $\phi_{\mathrm{M}}=0.30$ & $\phi_{\mathrm{F}}=0.001$ & \\
\hline Permeability & $\begin{array}{l}\mathrm{K}_{\mathrm{M}}=1.0 \times \\
10^{-15}\end{array}$ & $\begin{array}{l}\mathrm{K}_{\mathrm{F}}=1.0 \times \\
10^{-12}\end{array}$ & $\mathrm{~m}^{2}$ \\
\hline $\begin{array}{l}\text { Residual/initial } \\
\text { saturation }\end{array}$ & $\mathrm{S}_{\mathrm{Mr}}=0.2$ & $\mathrm{~S}_{\mathrm{Fr}}=0.2$ & \\
\hline $\begin{array}{l}\text { Coefficient of } \\
\text { permeability }\end{array}$ & $\mathrm{C}_{\mathrm{kM}}=1.0$ & $\mathrm{C}_{\mathrm{kF}}=0.2$ & $\mathrm{~Pa}$ \\
\hline $\begin{array}{l}\text { Coefficient of } \\
\text { capillary pressure }\end{array}$ & $\mathrm{C}_{\mathrm{pM}}=1.0 \times$ & $\begin{array}{l}\mathrm{C}_{\mathrm{pF}}=1.0 \times \\
10^{3}\end{array}$ & \\
\hline Saturation at well & $\mathrm{S}_{0}=0.279$ & & $\mathrm{~Pa}$ \\
\hline Fluid viscosity & $\mu_{\mathrm{w}}=1.0 \times 10^{-3}$ & $\mathrm{~kg} / \mathrm{m}^{3}$ \\
\hline Fluid density & $\mathrm{P}_{\mathrm{w}}=1,000$ & $\mathrm{~m}$ \\
\hline Wellibore radius & $\mathrm{r}_{\mathrm{w}}=0.1$ & \\
\hline
\end{tabular}


Table 4-3 Parameters used in the comparison with laboratory testing results (Kazemi, 1979)

\begin{tabular}{|c|c|c|}
\hline Parameter & Value & Unit \\
\hline Fracture aperture & $\mathrm{b}=0.0003$ & $\mathrm{~m}$ \\
\hline Fracture porosity & $\phi_{\mathrm{F}}=1.0$ & \\
\hline Matrix porosity & $\phi_{\mathrm{M}}=0.21$ & $\mathrm{~m}^{2}$ \\
\hline Absolute fracture permeability & $\mathrm{k}_{\mathrm{F}}=1 \times 10^{-11}$ & $\mathrm{~m}^{2}$ \\
\hline Absolute matrix permeability & $\mathrm{k}_{\mathrm{M}}=4,23 \times$ & \\
\hline Water density & $10^{-13}$ & $\mathrm{~kg} / \mathrm{m}^{3}$ \\
\hline Water viscosity & $\rho_{\mathrm{w}}=1,000$ & $\mathrm{~Pa} \bullet \mathrm{s}$ \\
\hline Oil (diesel) density & $\mu_{\mathrm{w}}=1 \times 10^{-3}$ & $\mathrm{~kg} / \mathrm{m}^{3}$ \\
\hline Oil (diesel) viscosity & $\rho_{\mathrm{w}}=828$ & $\mathrm{~Pa} \bullet \mathrm{s}$ \\
& $\mu_{\mathrm{w}}=4.6 \times 10^{-}$ \\
& & \\
\hline Residual fracture water & $\mathrm{S}_{\mathrm{wr}, \mathrm{F}}=0.10$ & \\
\hline saturation & & \\
\hline Residual matrix water saturation & $\mathrm{S}_{\mathrm{wr}, \mathrm{M}}=0.20$ & $\mathrm{~m} / \mathrm{d}$ \\
\hline Residual fracture oil saturation & $\mathrm{S}_{\mathrm{or}, \mathrm{F}}=0.0001$ & \\
\hline Initial fracture water saturation & $\mathrm{S}_{\mathrm{wi}, \mathrm{F}}=0.00$ & \\
\hline Initial matrix water saturation & $\mathrm{S}_{\mathrm{wi}, \mathrm{M}}=0.00$ & $\mathrm{q}=2.568 \times$ \\
\hline Water injection rate & $10^{-5}$ & \\
\hline
\end{tabular}




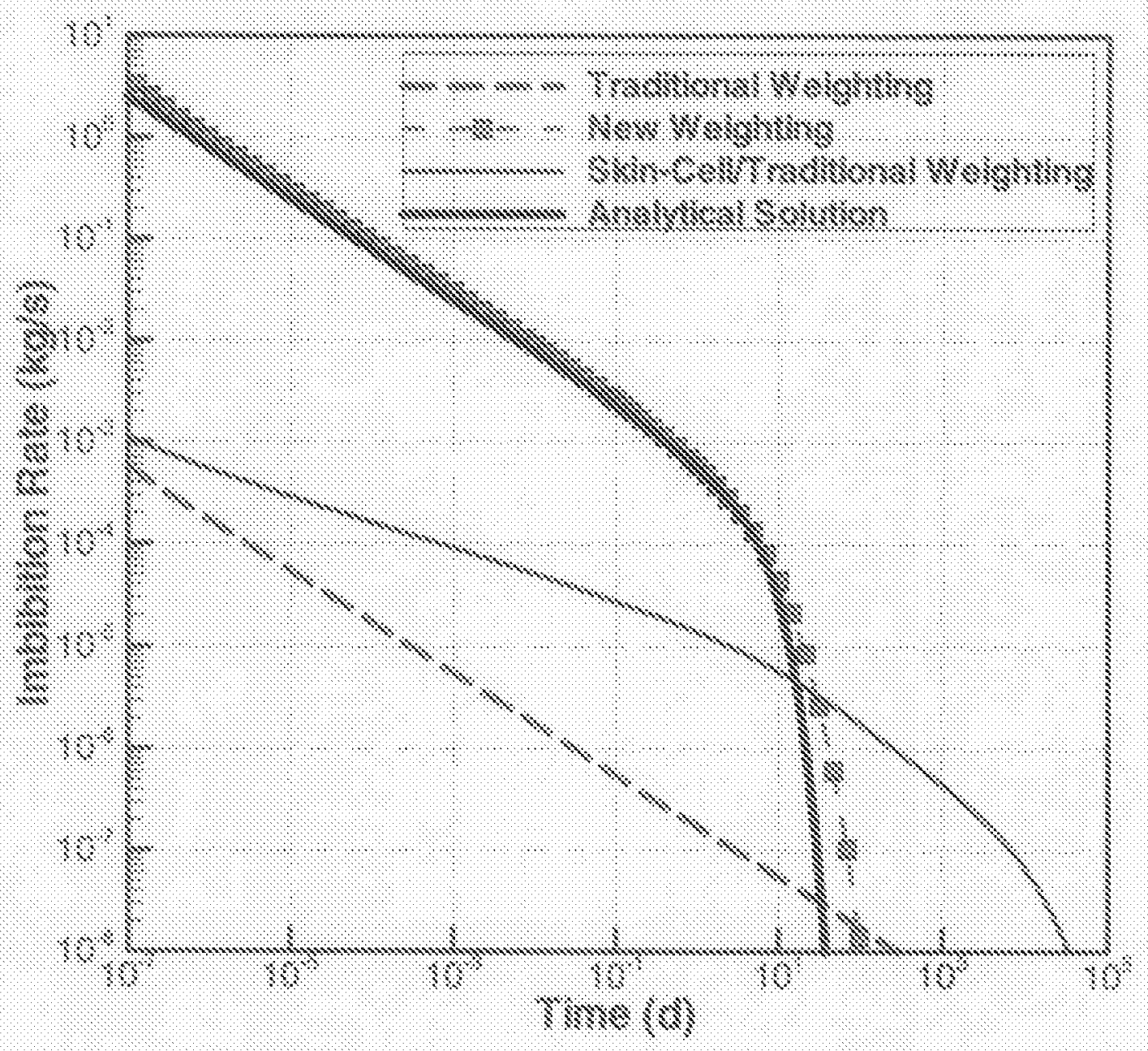

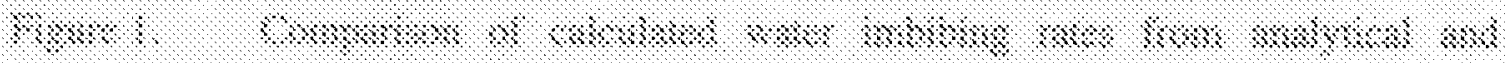

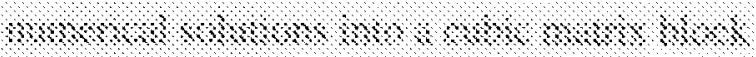




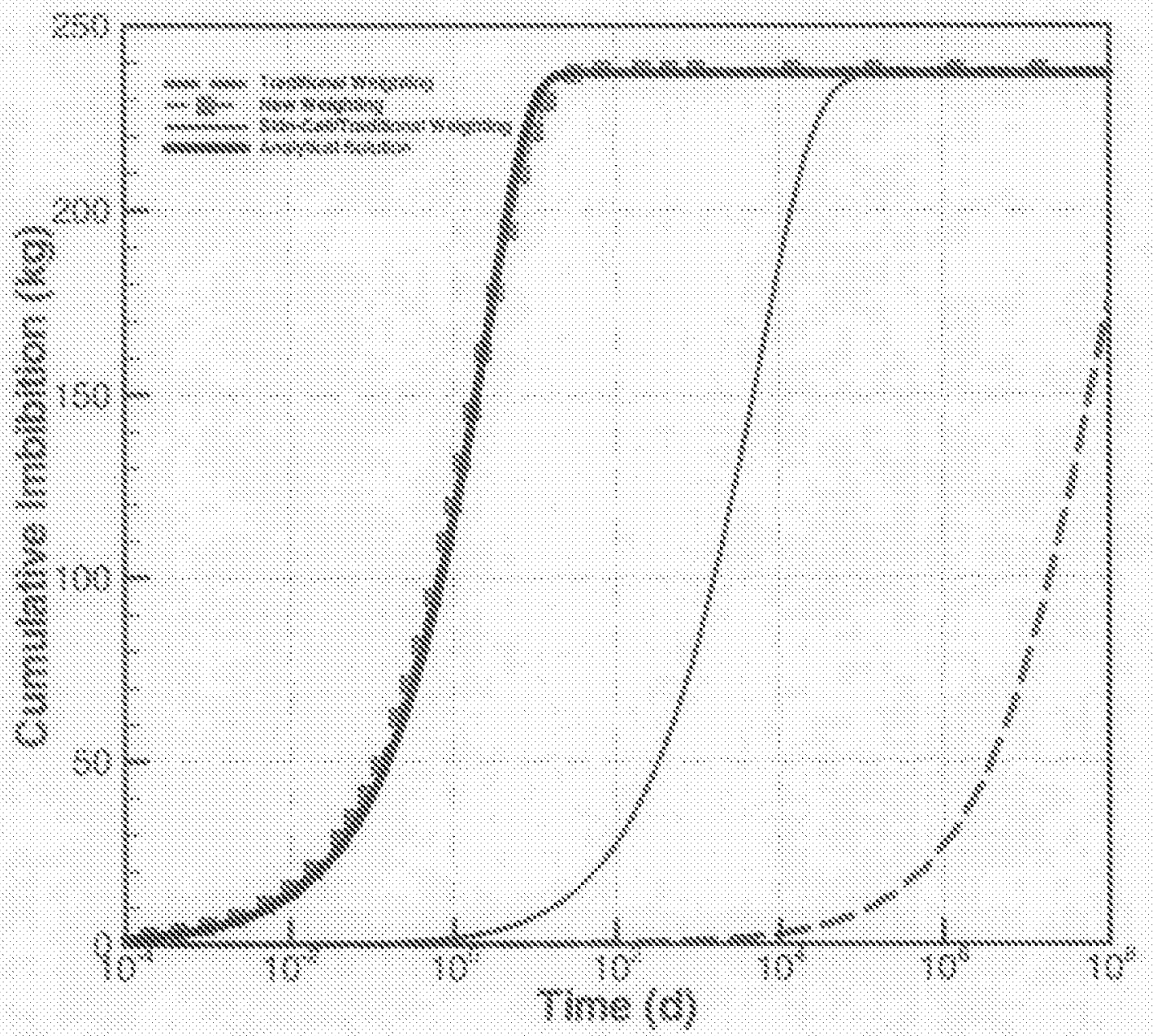

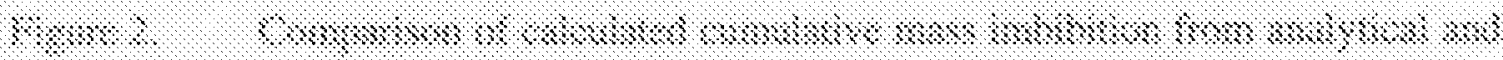

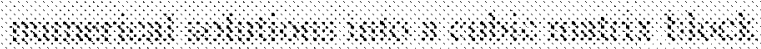




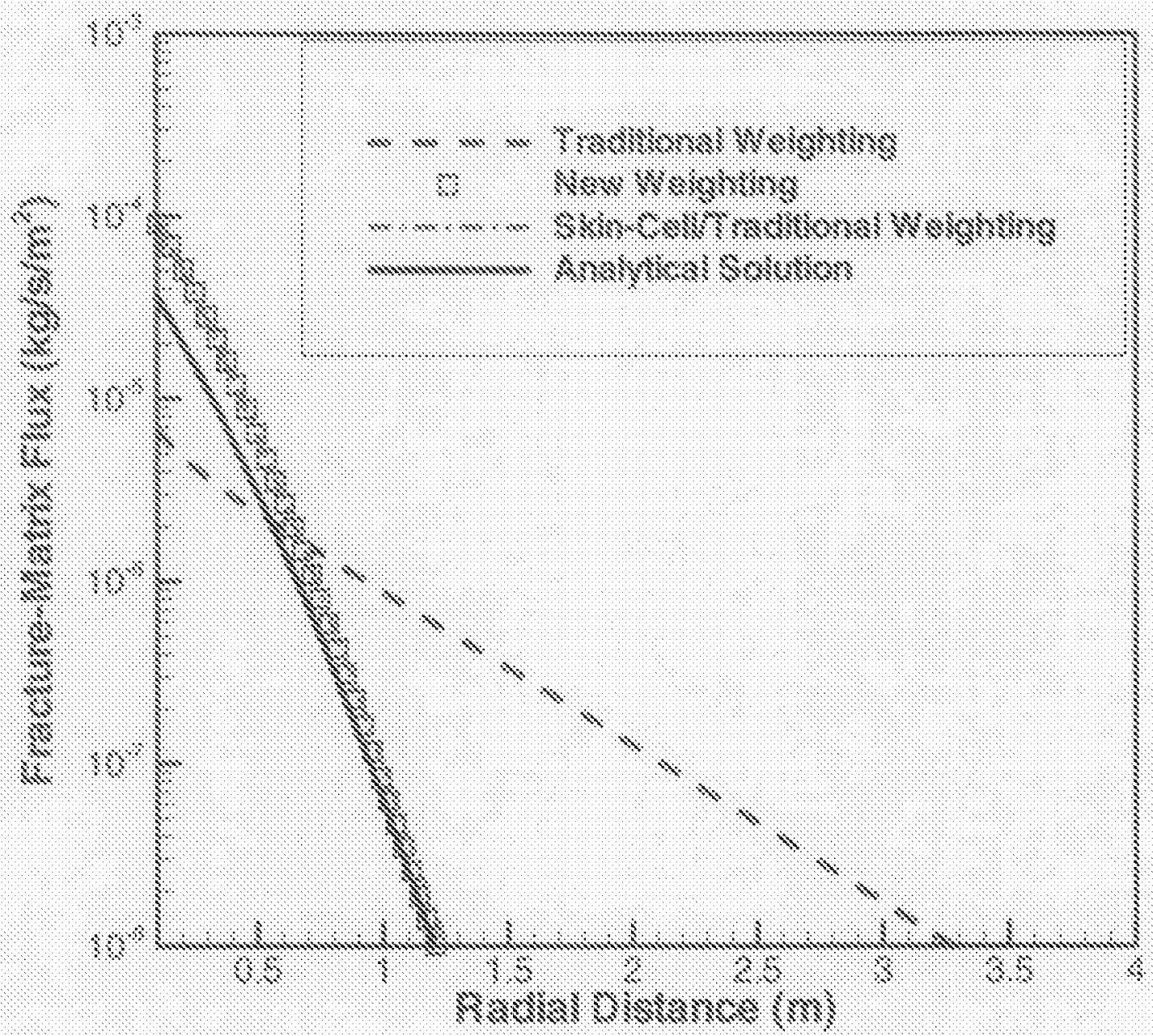

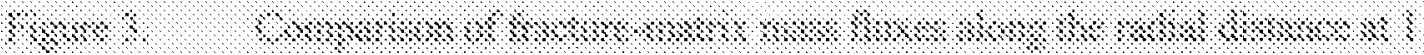

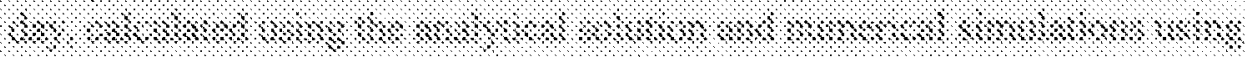

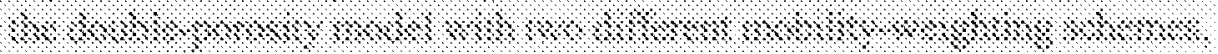

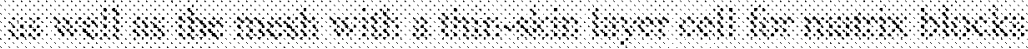




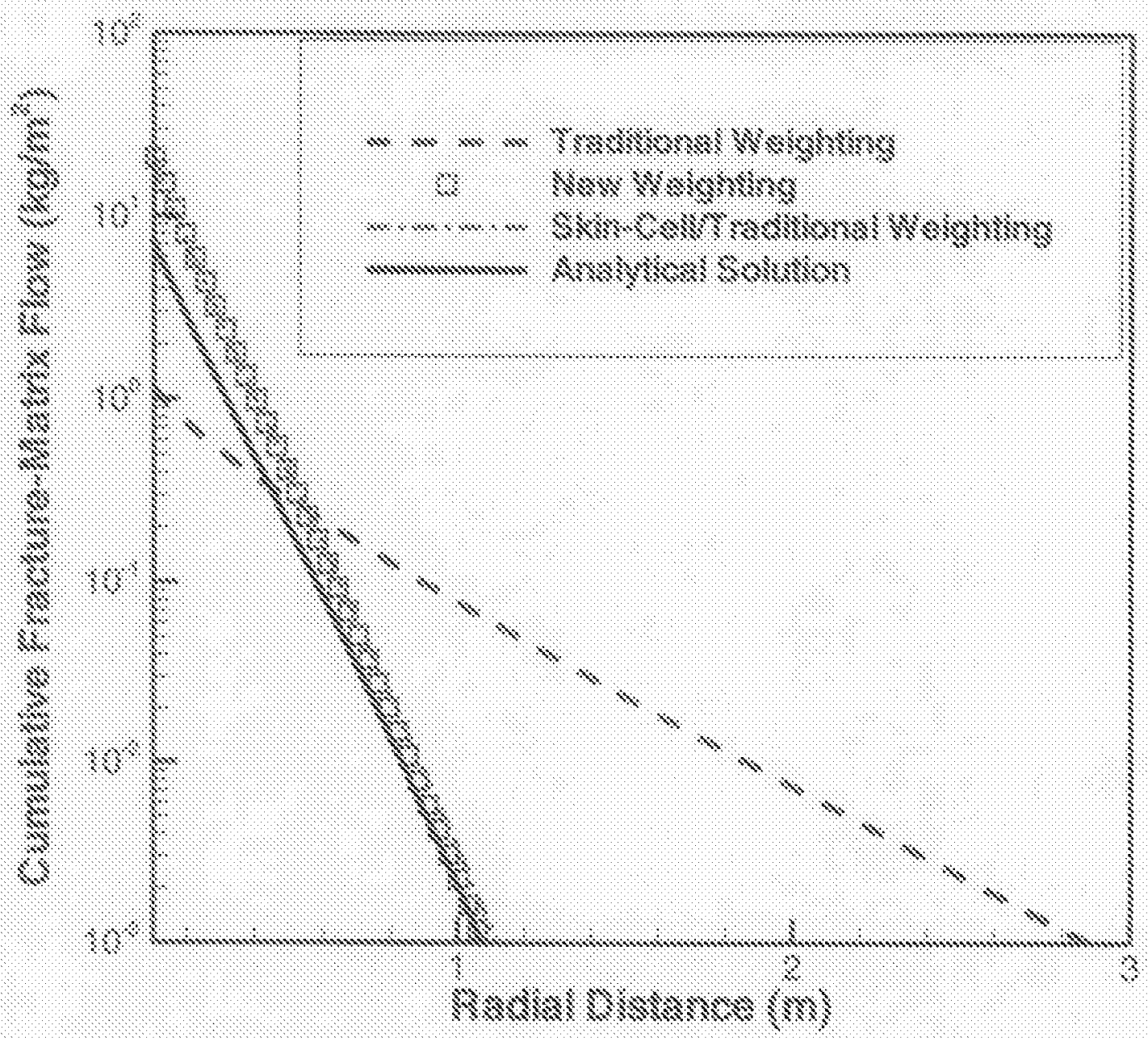

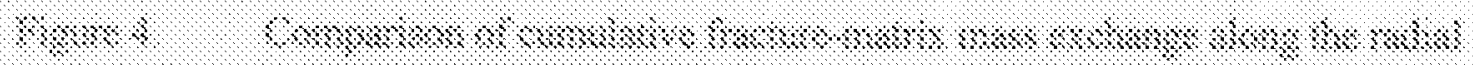

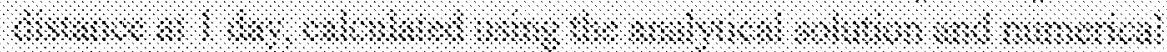

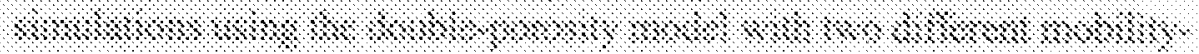
* \%०\%: 


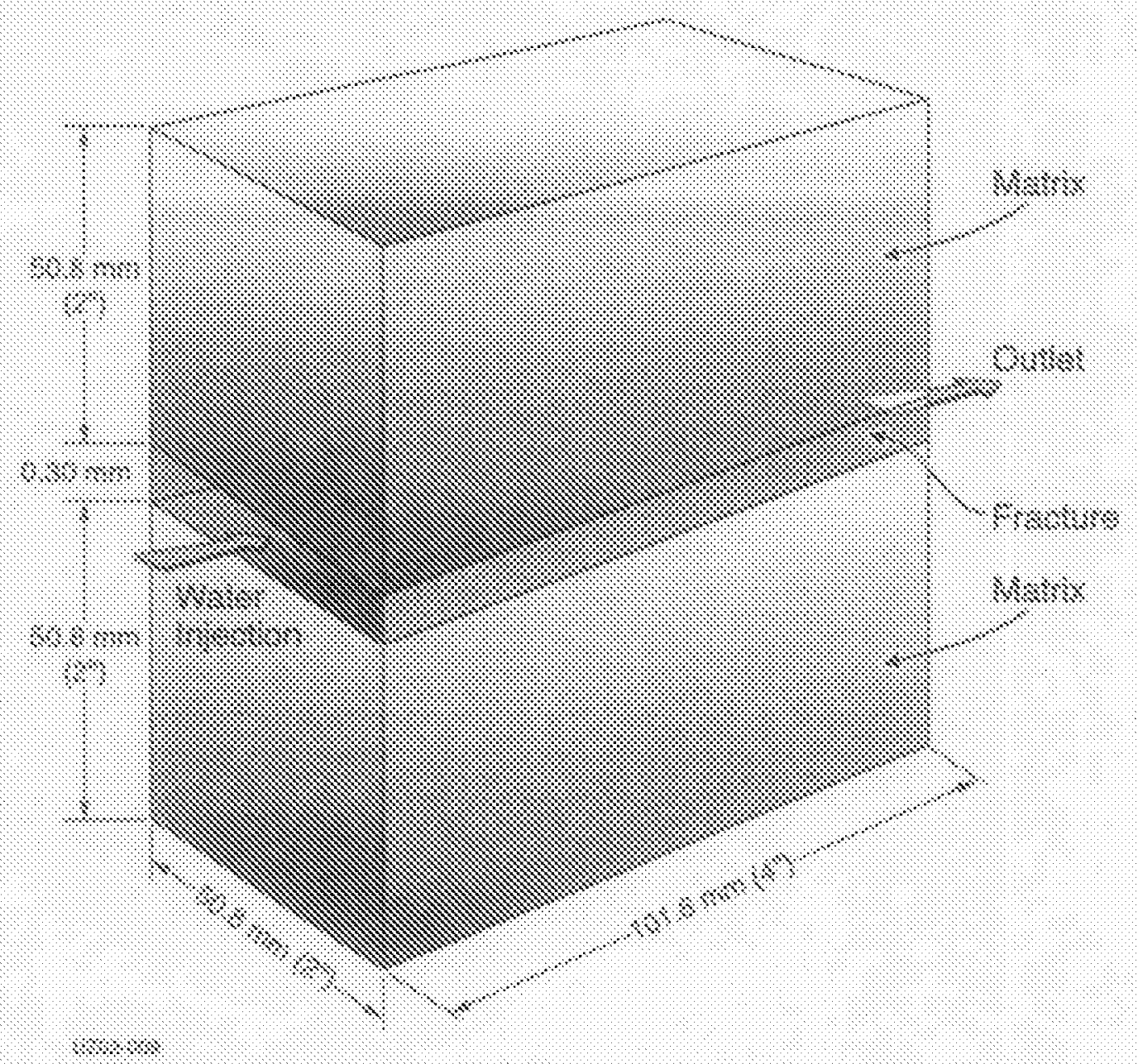

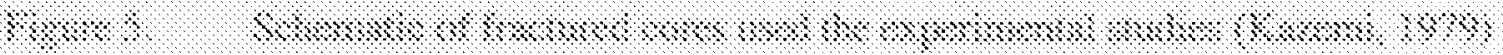




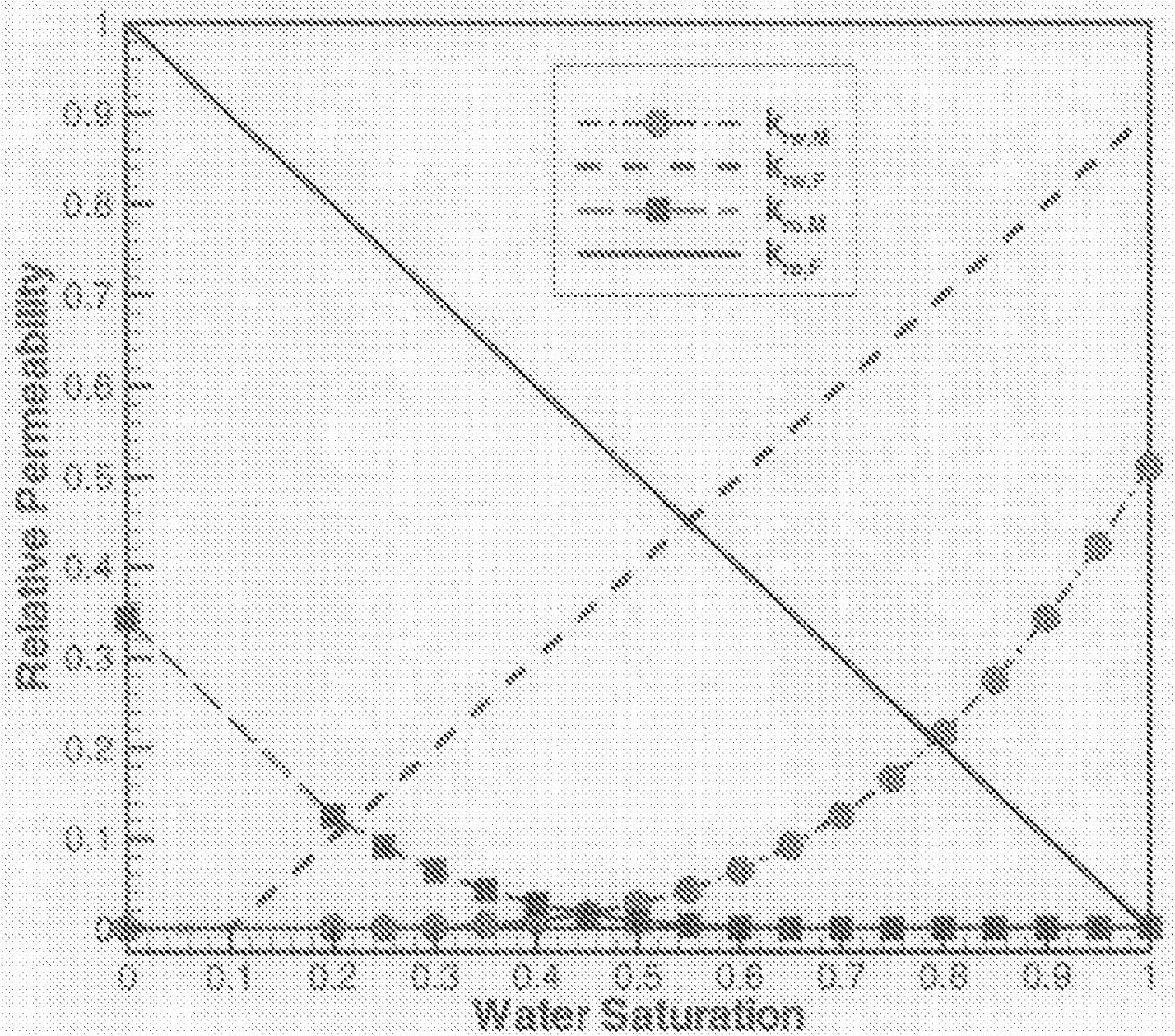

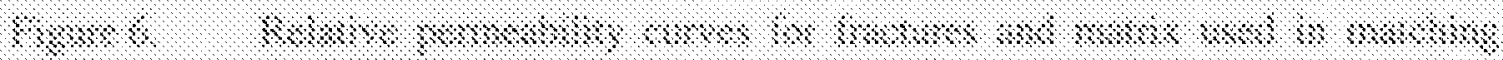

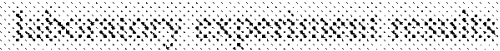




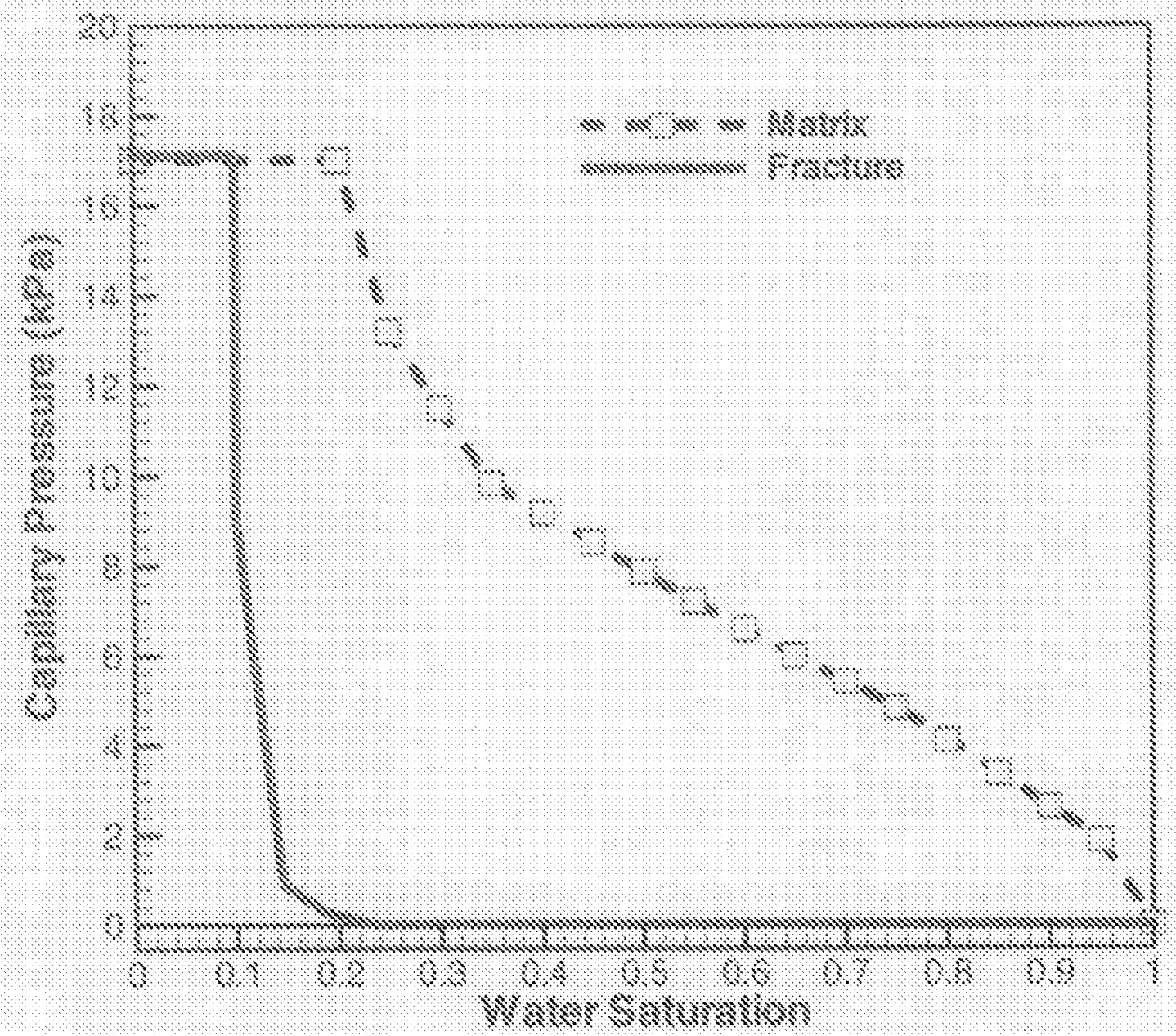

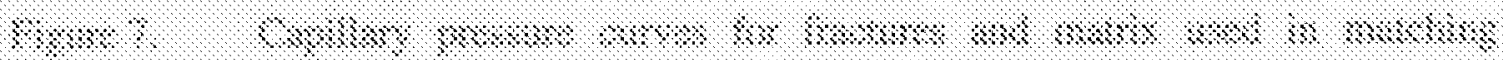

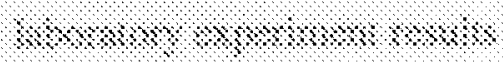




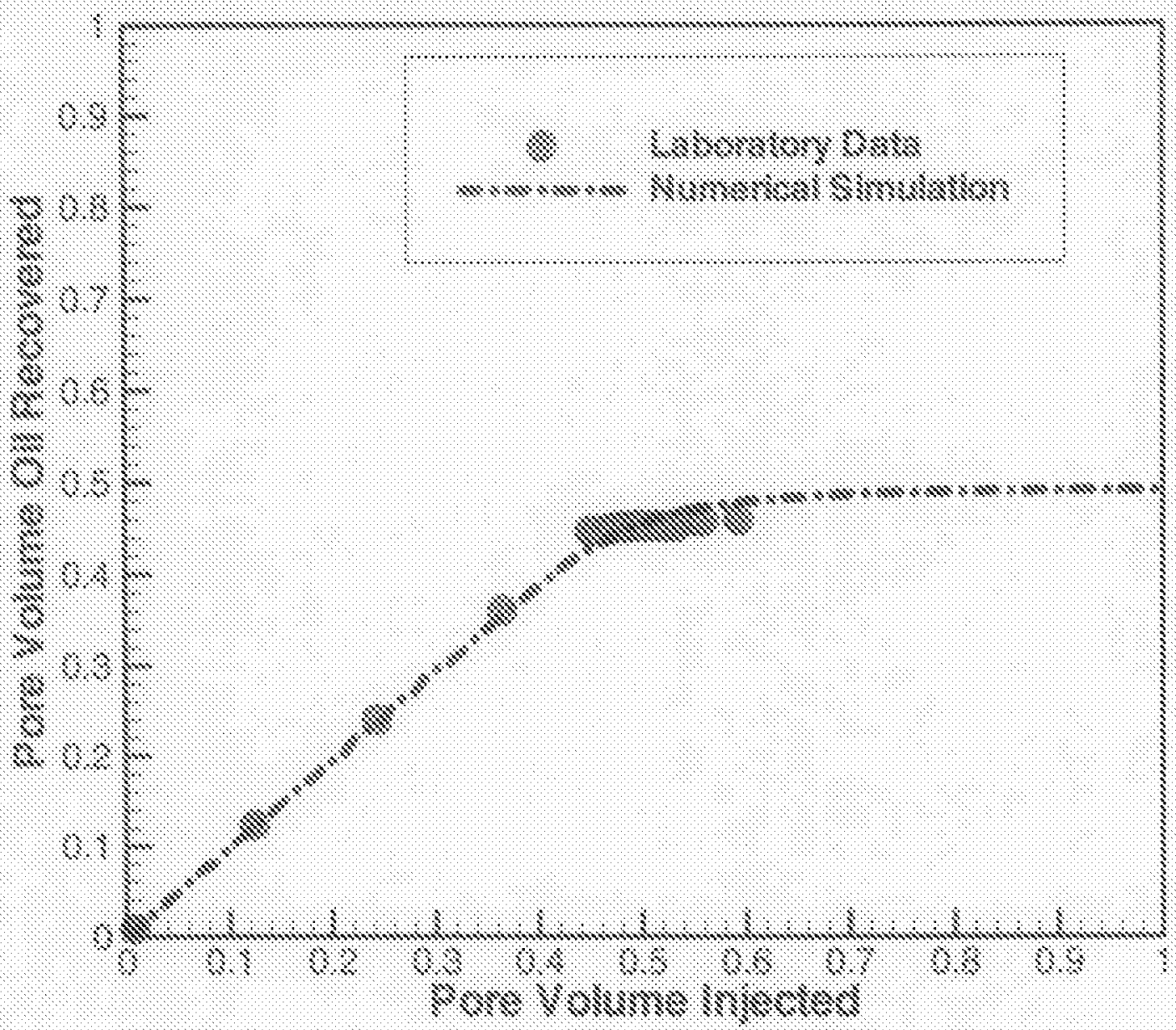

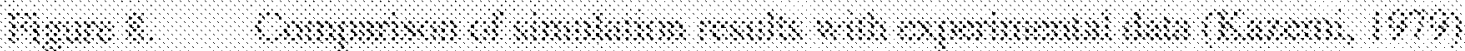




\title{
A Physically Based Approach for Modeling \\ Multiphase Fracture-Matrix Interaction in Fractured Porous Media
}

MOL. 20040630.0769

\author{
Yu-Shu Wu, Lehua Pan, and Karsten Pruess
}

\begin{abstract}
Modeling fracture-matrix interaction within a complex multiple phase flow system is a key issue for fractured reservoir simulation. Commonly used mathematical models for dealing with such interactions employ a dual- or multiple-continuum concept, in which fractures and matrix are represented as overlapping, different, but interconnected continua, described by parallel sets of conservation equations. The conventional singlepoint upstream weighting scheme, in which the fracture relative permeability is used to represent the counterpart at the fracture-matrix interface, is the most common scheme by which to estimate flow mobility for fracture-matrix flow terms. However, such a scheme has a serious flaw, which may lead to unphysical solutions or significant numerical errors. To overcome the limitation of the conventional upstream weighting scheme, this paper presents a physically based modeling approach for estimating physically correct relative permeability in calculating multiphase flow between fractures and the matrix, using continuity of capillary pressure at the fracture-matrix interface. The proposed approach has been implemented into two multiphase reservoir simulators and verified using analytical solutions and laboratory experimental data. The new method is demonstrated to be accurate, numerically efficient, and easy to implement in dual- or multiple-continuum models.
\end{abstract}

Key Words: Naturally fractured reservoir, double-porosity model, dual-continuum model, fracture-matrix interaction, relative permeability 


\section{Introduction}

Since the 1960s, significant progress has been made in numerical modeling of flow and transport processes in fractured rock. Research efforts, driven by the increasing need to develop petroleum and geothermal reservoirs, other natural underground resources, and to resolve subsurface contamination problems, have developed many numerical modeling approaches and techniques (Barenblatt et al., 1960; Warren and Root, 1963; Kazemi, 1969; Pruess and Narasimhan, 1985).

Mathematical modeling approaches in general rely on continuum approaches and involve developing conceptual models, incorporating the geometrical information of a given fracture-matrix system, setting up mass and energy conservation equations for fracture-matrix domains, and then solving discrete nonlinear algebraic equations. Most computational effort is consumed in solving the governing equations that couple multiphase fluid flow with other physical processes either analytically or numerically. The key issue for simulating flow in fractured rock, however, is how to handle facturematrix interaction under different conditions (involving multiple phase flow). This is because the fracture-matrix interaction distinguishes the flow through fractured porous media from the flow through heterogeneous single-porosity porous media. Note that many literature studies deal only with pure fracture media without fracture-matrix interaction by ignoring the effects of low-permeability matrix rocks. In those cases, the fractures can be treated as part of a classical single-porosity medium, which is not dealt with in this work.

To model fracture-matrix interaction, modelers have developed and applied many different conceptual models and modeling approaches as summarized in Berkowitz (2002). Commonly used mathematical methods include: (1) an explicit discrete-fracture and matrix model (e.g., Snow, 1969; Stothoff and Or, 2000), (2) the dual-continuum method, including double- and multiporosity, dual-permeability, or the more general "multiple interacting continua"' (MINC) method (e.g., Barenblatt et al., 1960; Warren and Root, 1963; Kazemi, 1969; Pruess and Narasimhan, 1985; Wu and Pruess, 1988), and (3) the effective-continuum method (ECM) (e.g., Wu, 2000a). 
The explicit discrete-fracture approach is, in principle, a rigorous model. However, the actual application of this method is currently limited because of the computational intensity involved as well as the lack of detailed knowledge of fracture and matrix geometric properties and their associated spatial distributions at a given site. On the other hand, the dual-continuum method is conceptually simpler and computationally much less demanding than the discrete-fracture approach, and is able to handle fracture-matrix interaction more easily than the discrete-fracture model. For these reasons, the dualcontinuum approach has been used as the main approach for modeling fluid flow, heat transfer, and chemical transport through fractured reservoirs (e.g., Wu et al., 1999).

Dual-continuum approaches include the classical double-porosity model (Barenblatt et al., 1960; Warren and Root, 1963), the dual-permeability concept, and the more rigorous dual-continuum generalization of the MINC (Pruess and Narasimhan, 1985) for modeling flow in fractured porous media. In the double-porosity model, a flow domain is composed of matrix blocks with low permeability, embedded in a network of interconnected fractures. Global flow and transport in the formation occur only through the fracture system, conceptualized as an effective continuum. This model treats matrix blocks as spatially distributed sinks or sources to the fracture system without accounting for global matrix-matrix flow. In comparison, the MINC concept (Pruess and Narasimhan, 1985) is able to describe gradients of pressures, temperatures, or concentrations between fractures and matrix systems and inside the matrix-by further subdividing individual matrix blocks with one- or multidimensional strings of nested meshes. Consequently, the MINC model in general provides a better numerical approximation for transient fracture-matrix interactions than the double-porosity model.

Because of its computational efficiency and its ability to match many types of laboratory- or field-observed data simultaneously (e.g., Kazemi, 1979; Wu et al., 1999), the dual-continuum model has perhaps been the most widely used method in petroleum and geothermal engineering and groundwater hydrogeology, and it has also been implemented in many commercially available reservoir simulators.

In numerical modeling of fracture-matrix flow, one of the critical issues is how to estimate flow mobility at the fracture-matrix interface. In conventional simulation 
practice, especially in petroleum reservoir simulation, the fully upstream weighting scheme (or simply upstream weighting or upwinding) is routinely used (e.g., Aziz and Settari, 1979). As a result, the fracture relative permeability is commonly selected in estimating such mobility when local flow is towards the matrix. However, this scheme is physically incorrect, because of the inherent anisotropy of the fracture-matrix medium at this scale. The fracture relative permeability functions are properties for flow along fractures, determined independently from matrix flow, for example, by laboratory studies (e.g., Persoff and Pruess, 1995). In general, fracture-matrix flow (or interaction) occurs perpendicular to fracture planes, which is controlled mainly by matrix flow properties. Therefore, the fracture relative permeability used in this case is physically incorrect and may lead to unphysical solutions or significant numerical errors.

The objective of this study is to develop a physically based upstream weighting scheme for determining relative permeability functions that can be generally applicable to calculating multiphase flow between fractures and the rock matrix using a dualcontinuum concept. Specifically, the proposed mobility-weighting approach has been implemented into two multiphase reservoir simulators to demonstrate its application. In addition, we attempt to verify the proposed weighting scheme by using two analytical solutions and published laboratory results. The new method is shown to be accurate, numerically efficient, and easy to implement in existing dual- or multiple-continuum models in reservoir simulators.

\section{Mathematical Formulation}

In the dual-continuum approach, multiphase flow processes in fractured rock are described separately, using a doublet of governing equations for the two continua: fracture and matrix. This conceptualization results in a set of partial differential equations for flow in either continuum, which are in the same foen as that for a single porous medium. In this work, the multiphase flow system, assumed in an isothermal, fractured porous formation, consists of three phases: gas (air), water, and NAPL (or oil), in which two-phase flow or the Richards' equation (1931) is considered as a special case. Although 
each of the three phases contains a number of components, they are treated here as a single "pseudo-component" with averaged properties of the fluids. In addition, the three fluid components (gas, water, and NAPL) are assumed to be present only in their associated phases. Each phase flows in response to its pressure gradients, gravitational, and capillary forces, according to the multiphase extension of Darcy's law.

In an isothermal system containing three mass components, three mass balance equations are needed to describe flow and transport in the fracture and matrix blocks. For flow of phase $\beta$ ( $\beta=\mathrm{g}$ for gas, $\beta=\mathrm{w}$ for water, and $\beta=\mathrm{o}$ or $\mathrm{n}$ for NAPL),

$$
\frac{\partial}{\partial t}\left(\phi S_{\beta} \rho_{\beta}\right)=-\nabla \cdot\left(\rho_{\beta} \mathbf{v}_{\beta}\right)+q_{\beta}
$$

where the Darcy velocity of phase $\beta$ is defined by:

$$
\mathbf{v}_{\beta}=-\frac{k k_{r \beta}}{\mu_{\beta}}\left(\nabla P_{\beta}-\rho_{\beta} g \nabla D\right)
$$

In Equations (2-1) and (2-2), $\rho_{\beta}$ is the density of phase $\beta$ under reservoir conditions; $\phi$ is the effective porosity of the medium; $\mu_{\beta}$ is the viscosity of phase $\beta ; S_{\beta}$ is the saturation of phase $\beta ; P_{\beta}$ is the pressure of phase $\beta ; q_{\beta}$ is the sink/source term of phase (component) $\beta$ per unit volume of formation; $g$ is gravitational acceleration; $\mathrm{k}$ is the absolute/intrinsic permeability of the formation; $\mathrm{k}_{\mathrm{r}}$ is relative permeability to phase $\beta$; and $D$ is depth from a datum.

The governing equation of mass balance for three-phase fluids, Equation (2-1), needs to be supplemented with constitutive equations, which express all the secondary variables and parameters as functions of a set of primary variables of interest. In particular, the relationships include relative permeability and capillary pressure functions as well as other PVT data. In addition, the initial and boundary conditions of the system are also needed to complete the description of multiphase flow through fractured or porous media. 


\section{Numerical Formulation}

\subsection{Discrete Equations}

The multiphase flow equations, as discussed in Section 2, have been implemented into a general-purpose two-phase code TOUGH2 (Pruess et al., 1999) and a three-phase reservoir simulator MSFLOW (Wu, 2000b). As implemented numerically, Equation (2-1) is discretized in space using an integral finite-difference or control-volume scheme for a porous and/or fractured medium. The time discretization is carried out with a backward, first-order, finite-difference scheme. The discrete nonlinear equations for water, NAPL, and gas flow at node $i$ are written as follows:

$$
\left\{\left(\phi S_{\beta} \rho_{\beta}\right)_{i}^{n+1}-\left(\phi S_{\beta} \rho_{\beta}\right)_{i}^{n}\right\} \frac{V_{i}}{\Delta t}=\sum_{j=\eta_{i}} F_{\beta, i j}^{n+1}+Q_{\beta i}^{n+1}
$$

(for $\beta=\mathrm{g}$, w and o).

where superscript $\mathbf{n}$ denotes the previous time level; $n+1$ is the current time level; $V_{i}$ is the volume of element $\mathrm{i}$ (porous or fractured block); $\Delta t$ is time step size; $\eta_{\mathrm{i}}$ contains the set of neighboring elements (j) (porous or fractured) to which element $i$ is directly connected; $F_{\beta, i j}$ is the mass flow term for phase $\beta$ between elements $i$ and $j$; and $Q_{\beta_{i}}$ is the mass sink/source term at element $i$, of phase $\beta$.

The "flow" term $\left(\mathrm{F}_{\beta, \mathrm{ij}}\right)$ in Equation (3-1) for single-phase, Richards', or multiphase flow is described by a discrete version of Darcy's law. This is the mass flux of fluid phase $\beta$ along the connection is given by

$$
\mathrm{F}_{\beta, \mathrm{ij}}=\lambda_{\beta, \mathrm{j} j+1 / 2} \gamma_{\mathrm{ij}}\left[\psi_{\beta \mathrm{j}}-\psi_{\beta \mathrm{i}}\right]
$$

where $\lambda_{\beta_{i j+1 / 2}}$ is the mobility term to phase $\beta$, defined as 
$\lambda_{\beta, i j+1 / 2}=\left(\frac{\rho_{\beta} k_{r \beta}}{\mu_{\beta}}\right)_{i j+1 / 2}$

and subscript $i j+1 / 2$ denotes a proper averaging or weighting of properties at the interface between two elements $i$ and $j$, discussed in the sections below, and $k_{r} \beta$ is the relative permeability to phase $\beta$. In Equation (3-2), $\gamma_{i j}$ is transmissivity and is defined differently for finite-difference or finite-element discretization. If the integral finite-difference scheme (Pruess et al., 1999) is used, the transmissivity is calculated as

$$
\gamma_{i j}=\frac{A_{i j} k_{i j+1 / 2}}{d_{i}+d_{j}}
$$

where $A_{i j}$ is the common interface area between connected blocks or nodes $i$ and $j ; d_{i}$ is the distance from the center of block $i$ to the interface between blocks $i$ and $j$; and $k_{i j+1 / 2}$ is an averaged (such as harmonic weighted) absolute permeability along the connection between elements $i$ and $j$,

The flow potential term in Equation (3-2) is defined as

$$
\psi_{\beta \mathrm{i}}=\mathrm{P}_{\beta \mathrm{i}}-\rho_{\beta, \mathrm{j} j+1 / 2} g D_{\mathrm{i}}
$$

where $D_{i}$ is the depth to the center of block $i$ from a reference datum.

Discrete Equation (3-1) has the same form regardless of the dimensionality of the model domain, i.e., it applies to one-, two-, or three-dimensional analyses of flow through fractured or porous media. In our numerical model, Equation (3-1) is written in a residual form and is solved using Newton/Raphson iteration. 


\subsection{Handling Fractured Media}

The technique used in this work for handling multiphase flow through fractured rock follows the dual-continuum methodology (Warren and Root, 1963; Pruess and Narasimhan, 1985). This method treats fracture and matrix flow and interactions using a multi-continuum numerical approach, including the double- or multiporosity method, the dual-permeability method, and the more general MINC method (Pruess and Narasimhan, 1985). It can be shown that the same continuum concept is also applicable to multiphase flow though a discrete fracture network.

The multiphase flow formulation, Equations (2-1) and (3-1), is applicable to both single-continuum and multi-continuum media. Using the dual-continuum concept, Equations (2-1) and (3-1) can be used to describe multiphase flow both in fractures and inside matrix blocks, as well as fracture-matrix interaction. However, special attention needs to be paid to treating fracture-matrix flow. The flow between fractures and the matrix is still evaluated using Equation (3-2); however, the transmissivity for the fracturematrix flow is given by

$$
\gamma_{\mathrm{ij}}=\frac{\mathrm{A}_{\mathrm{FM}} \mathrm{k}_{\mathrm{M}}}{l_{F M}},
$$

where $A_{F M}$ is the total interfacial area between fractures and the matrix of elements $i$ and $\mathrm{j}$ (one of them is a fracture and the other a matrix block); $\mathrm{k}_{\mathrm{M}}$ is the matrix absolute permeability along the fracture-matrix connection; and $l_{F M}$ is a characteristic distance for flow crossing fracture-matrix interfaces, which can be determined for idealized 1-D, 2-D and 3-D dimensional rectangular matrix blocks when using the double-porosity model (Warren and Root, 1963). 


\subsection{Mobility Weighting Scheme}

The appropriate spatial weighting scheme for averaging flow properties, such as the mobility of Equation (3-3) in a heterogeneous formation has been much debated in reservoir simulation and groundwater-modeling literature (Peaceman, 1977; Huyakorn and Pinder, 1983). Single-point or fully upstream weighting has been the exclusive approach for averaging mobility or relative permeability in calculating flow term, using a discrete Darcy's law for multiphase flow in heterogeneous petroleum reservoirs (Aziz and Settari, 1979). The reasons behind the early application of the conventional upstream weighting scheme for relative permeability were based on several physical arguments, such as the need for upstream weighting to initialize imbibition into completely dry rock. In addition, the upstream weighting approach was found to be necessary to avoid incorrect solutions in immiscible displacement (hyperbolic) problems (Aziz and Settari, 1979).

Recently, several theoretical studies (Forsyth et al., 1995; Forsyth and Kropinski, 1997) have shown that the upstream weighting scheme, if used with the control-volume discretization of the Richards' equation, will satisfy monotonicity conditions regardless of time step or mesh size. It will guarantee that converged numerical solutions are physically correct, while other weighting schemes, such as central weighting, may converge to an incorrect, unphysical solution (Forsyth and Kropinski, 1997). However, determining flow along fracture-matrix connections (i.e., flow across fracture-matrix interfaces in the direction perpendicular to fracture planes) is different from fracture-fracture flow and the conventional upstream weighting scheme may no longer be applicable. This is because fracture relative permeability functions are fracture flow properties describing flow along fractures, determined independently from matrix flow. Conversely, fracture-matrix flow or interaction normally occurs along the directions perpendicular to fractures and is largely controlled by matrix properties or by flow resistance within the matrix block. The physical inconsistency in selecting fracture relative permeability for calculating fracturematrix flow may lead to unphysical solutions or significant numerical errors. 
To overcome these limitations, this paper presents a modified upstream weighting scheme for fracture-matrix interaction. This new scheme is based on the principle that the capillary pressure is continuous at the fracture-matrix interface, and the assumption that there is instantaneous local equilibrium in pressure for each phase on the matrix surface between fracture and matrix systems. This should hold true for most subsurface fractured reservoirs, because fracture aperture is normally very small and fracture lateral boundaries are defined by matrix surfaces. Any dynamic changes in fractures, such as capillary pressures, could be instantaneously equilibrated locally with that at contacted matrix surfaces. As a result, the matrix relative permeability at the matrix surface can be readily determined as a function of fracture capillary pressure, or the matrix saturation corresponding to that fracture capillary pressure. Therefore, the new scheme, when the upstream direction for fracture-matrix flow is at the fractures, uses the matrix relative permeability function (instead of the fracture relative permeability function, as in the conventional upstream weighting scheme) to calculate the mobility. Physically, this is equivalent to evaluating flow through the fracture-matrix interface into the matrix with the effective matrix permeability at that interface, obviously a more reasonable approach. The proposed weighting scheme is still dependent on the upstream fracture condition, and therefore does not lose the advantages of upstream schemes. In addition, in case fracturematrix flow is from matrix to fractures, such as in a situation of drainage or flow between globally connected fractures or along global or local matrix-matrix connections, the conventional upstream weighting scheme should still be used. We call this hybrid scheme capillary pressure-based weighting or physically based upstream weighting.

Within the context of the dual-continuum concept, the proposed approach can be applied to different matrix discretizations, such as double-porosity, dual-permeability, or MINC grids. We have implemented the proposed physical upstream weighting scheme into two multidimensional reservoir simulators and conducted a series of numerical experiments with all the commonly used dual-continuum models. In all test cases, this new weighting scheme is found to work efficiently, similarly to using the traditional single-point upstream weighting, without numerical difficulties. Note that this new scheme should be applicable for discrete fracture-network models as well. 
Note that implementation of the new weighting scheme, though straightforward, requires modifying existing reservoir simulators. Alternatively, following the same physical reasoning as above, we can implement the scheme by creating a matrix mesh without modifying reservoir simulators. This requires generation of a thin skin-layer cell, with a tiny volume fraction, along the matrix surface for every matrix block. Then a simulation using a grid with skin-layer matrix discretization with traditional upstream weighting may achieve similar results to that of the physical upstream weighting of the new scheme, as long as the skin cell is sufficiently small. This is because it takes little time to equilibrate fractures with the matrix-surface skin cell, and fracture-matrix flow is equivalent to the flow between the matrix-surface skin cell and the inside or majority portion of the matrix block. This matrix-matrix flow is then treated correctly using the upstreamed matrix properties with the conventional weighting scheme. We show in the following sections that adding a thin skin-layer approach provides better results as compared to the traditional upstream weighting scheme in modeling fracture-matrix interaction. It should be mentioned, however, that adding a skin matrix layer in a doubleporosity grid increases the number of gridblocks by $50 \%$. How small the added skin cells should be needs to be determined by numerical experiments. In addition, the small volume of skin-layer cells reduces attainable time steps. These factors should be taken into account when applying these modeling approaches.

\section{Validation and Application Examples}

In an effort to examine and verify the proposed mobility-weighting scheme in this section, we present three validation and application examples. The proposed physical upstream weighting scheme has been implemented in the two reservoir simulators TOUGH2 (Pruess et al., 1999; Wu et al., 1996) and MSFLOW (Wu, 2000b), which are used in the following application examples. The first two examples compare numerical simulation results with analytical solutions for Richards' equation (Wu and Pan, 2003; 2004). The third case is to match published laboratory experiment results of water imbibition and displacement conducted on fractured cores (Kazemi, 1979). 


\subsection{Comparison with the Analytical Solution for Imbibition into a Single Matrix Block}

An analytical solution recently derived for water imbibition into an unsaturated cubic matrix block, using the Richards' equation (Wu and Pan, 2003), is applied here to examine numerical simulation results with the new mobility-weighting scheme. We select a 1-D spherical flow analytical solution in this study, which requires the following special forms of relative permeability $k_{\mathrm{ru}}$ and capillary pressure $P_{c}$,

$$
\mathrm{k}_{\mathrm{rw}}\left(\mathrm{S}_{\mathrm{w}}\right)=\mathrm{C}_{\mathrm{k}}\left(\overline{\mathrm{S}}_{\mathrm{w}}\right)^{\alpha}
$$

and

$$
P_{c}\left(S_{w}\right) \equiv P_{g}-P_{w}=C_{p}\left(\bar{S}_{w}\right)^{-\beta}
$$

where $\mathrm{P}_{\mathrm{g}}$ is a constant air (or gas) pressure, $\mathrm{C}_{k}$ and $\mathrm{C}_{\mathrm{p}}(\mathrm{Pa})$ are coefficients, $\alpha$ and $\beta$ are exponential constants, respectively, of relative permeability and capillary-pressure functions, and $\overline{\mathrm{S}}_{\mathrm{w}}$ is the normalized water saturation,

$$
\bar{S}_{w}=\frac{S_{w}-S_{w r}}{1-S_{w r}}
$$

with $\mathrm{S}_{\mathrm{wr}}$ being the residual water saturation. In addition, the relative permeability and capillary pressures are correlated by the following condition:

$$
\alpha=\beta+1
$$

To examine the new weighting scheme for imbibition into a matrix block, numerical simulations are performed using the TOUGH2 code, which incorporates the same relative 
permeability and capillary pressure functions, Equations (4-1) and (4-2), as required by the analytical solutions.

The example problem deals with transient water imbibition into a $1 \times 1 \times 1 \mathrm{~m}$ low initial water saturation matrix cube, which is surrounded by a uniform fracture network of three orthogonal sets with constant liquid saturation. The imbibition starts at $t=0$, owing to nonequilibrium in capillarity between the fracture and matrix systems, imposed as the boundary condition to the matrix surface. For comparison, the imbibition process is modeled by both the analytical and numerical solutions. In the analytical solution, the continuity condition in capillary pressure is imposed on the matrix surface, i.e., the matrix block surface is subject to a constant saturation condition. The numerical model uses one double-porosity grid and one MINC grid. The MNC grid subdivides the matrix cube into two elements, and the first matrix element is a tiny-volume, skin-layer cell with a volumetric fraction set at 0.0001 of the original matrix volume.

The fracture-matrix parameters used for the example are listed in Table 4-1. Note that in the numerical model, fracture relative permeability and capillary pressure functions are also needed, for which van Genuchten relations (1980) are selected. One the other hand, the analytical solution needs those for the matrix only, defined in Equations (4-1) and (4-2). The initial fracture water saturation is 0.012 , which corresponds to $S_{w}=0.99$ on the matrix surface. It should be mentioned that in this work, we are concerned mainly with multiphase exchange at fracture-matrix interfaces, not with detailed spatial distributions of saturation within fractures or the matrix. Specifically, we compare the results in terms of mass flux (or imbibition rate) and cumulative mass exchange (or imbibition) between the fracture and matrix systems, as shown in Figures 1 and 2.

Figure 1 presents the results of transient imbibition rates on the matrix surface, calculated from the analytical solution and numerical simulations using traditional upstream weighting, the proposed new physical upstream weighting, and skin-cell with traditional upstream weighting schemes. Comparison of the three numerical model results with the analytical solution in Figure 1 clearly indicates that numerical results with the new weighting scheme agree the best with the analytical solution during the entire transient imbibing period. The simulation results with the skin-layer cell in this case do not match the 
analytical result very well. In contrast, the simulation using the traditional upstream weighting scheme presents the worst comparison, with more than three orders of magnitude lower than the results of the analytical solution during the entire transient imbibition period. This is because the upstream fracture relative permeability, selected by the traditional approach, significantly underestimated the mobility term for fracture-matrix flow. In terms of cumulative imbibition, similarly, Figure 2 also shows that the proposed weighting approach matches the analytical results very well, while adding a skin layer in matrix discretization provides an intermediate result and the traditional weighting scheme gives the worst prediction.

Figures 1 and 2 show surprisingly good matches between the results of the proposed new scheme and the analytical solution. This is in part due to the use of log-log (Figure 1) or semi-log (Figure 2) scales in plots, as well as large errors introduced by the modeling results from the other two approaches. Actually, the new weighting scheme with doubleporosity discretization of the fracture-matrix system also introduces some numerical errors of $50 \%$ at the very early transient times of $10^{-7}$ days (Figure 1). Even larger errors appear at later times when the cumulative mass approaches $237 \mathrm{~kg}\left(=\phi_{M} \times V_{M} \times\left(0.99-S_{M i}\right) \times \rho_{W}=0.3\right.$ $\times 1 \times 0.79 \times 1,000$ ), which is at equilibrium with the proposed boundary condition, as shown in Figures 1 and 2. We could match the flux much better if we used more refined discretization for the matrix block in addition to the new weighting scheme (Wu and Pan, 2003).

\subsection{Comparison with the Analytical Solution for Transient Radial Flow through Unsaturated Fractured Formation}

This section further validates the new weighting scheme using a more realistic analytical solution (Appendix A), which describes the transient unsaturated fracturematrix interaction for radial flow through a uniform, radially infinite fractured formation, consisting of many matrix blocks and fracture sets.

This verification problem addresses transient flow through a fully penetrating well into a uniform, horizontal fractured formation, which is $10 \mathrm{~m}$ thick. The fractured formation 
consists of uniform, identical $1 \times 1 \times 1 \mathrm{~m}$ cubes of matrix blocks, surrounded by a uniform, 3-D fracture network. The geological model considered is identical to the Warren and Root (1963) conceptual model. Initially, both fracture and matrix are at dry conditions with both initial saturations equal to their residual values, respectively. A constant saturation of $S_{W}=0.279$ is imposed at the wellbore as the inner boundary condition in the beginning, and then water is sucked into the formation from the well immediately.

In the numerical model, a finite reservoir $\left(r_{e}=100 \mathrm{~m}\right)$ of $10 \mathrm{~m}$ thickness is used, and the disk-type formation is discretized into a one-dimensional radially symmetric (primary) grid. The radial domain from $r_{w}=0.1 \mathrm{~m}$ to distance $r_{e}=100 \mathrm{~m}$ is subdivided into 500 intervals, with $\Delta \mathrm{r}=0.005 \mathrm{~m}$ for the first 200 elements, and the rest of the domain is subdivided into 300 gridblocks following a logarithmic scale. Two numerical grids are generated, a regular double-porosity mesh and a MINC mesh with an added, thin-skin layer cell to matrix blocks (with a volumetric fraction of 0.0001 ). The double-porosity grid represents the matrix system by one mesh locally and is used for simulations with the traditional and new upstream weighting schemes, while the MINC mesh subgrids each matrix block with 2 nested cells, used for the added skin-cell case.

For this problem, fracture-matrix rock and fluid properties are given in Table 4-2. Numerical simulations with different weighting schemes and grids are performed using the TOUGH2 code. The analytical solution (Appendix A) for fracture-matrix flux is evaluated using a numerical Laplace inversion (Wu and Pan, 2004). Three numerical simulations were completed for this problem. The first two use the double-porosity grid with the two mobility-weighting schemes, i.e., the traditional single-point upstream weighting and the proposed physical upstream weighting, and the third simulation is based on the MINC mesh with the thin-skin layer cell on matrix surfaces, using the traditional upstream weighting.

Figure 3 presents fracture-matrix flux distributions along the radial distance at one day, simulated by the analytical and three numerical modeling results. Note that the physical process simulated in this example is extremely nonlinear and dynamic. The initial liquid saturations are at residual values for both fracture and matrix systems. At the beginning, the boundary saturation for fractures at the wellbore jumps to a constant $=$ 
0.279) (flow rate at the well thus becomes infinitely large). Once imbibed into the fractures near the well, the liquid will be drawn by two forces in two different directions, flowing along fractures away from the well, and simultaneously imbibing into dry matrix blocks. A comparison shown in Figure 3 indicates that both the proposed weighting scheme and the skin-cell model do a much better job in matching the actual distribution of fracturematrix mass flux, as determined by the analytical solution, than the traditional weighting, double-porosity model. This implies that (in this case) the approaches for applying the physical upstream weighting concept or adding a skin layer better capture these physical processes by selecting physically correct relative permeability in estimating fracture-matrix interaction. Figure 3 clearly shows that in general the traditional upstream weighting scheme, by selecting a fracture relative permeability function to calculate fracture-matrix flow, cannot match the analytical results. Near the well, both the proposed new scheme and adding a skin layer cause an overestimate in fracture-matrix mass flux; while near the front of water imbibition in fractures, away from the well, the result from the new weighting matches slightly better with the analytical solution.

Figure 4 shows a comparison between cumulative or net fracture-matrix mass exchange, calculated by the analytical solution, and the three numerical model results. Note that the cumulative flow of Figure 4 is not the net amount of the actual mass exchange at a distance of $r$ for the time. Rather this value represents a cumulative mass exchange at the location and the time if the fracture-matrix interface area were $1 \mathrm{~m}^{2}$, which is determined by integrating fracture-matrix fluxes over the time at the location (Appendix A). Therefore, the cumulative flow of Figure 4 reflects integrated or accumulated effects of fracture-matrix interaction for the problem. Similar to Figure 3 with fracture-matrix fluxes, Figure 4 indicates that adapting the new weighting scheme or adding a skin-cell also matches cumulative mass exchanges and their spatial distributions well, while the traditional weighting again leads to significant errors in estimating cumulative results.

Many more numerical experiments and comparisons (using different parameter sets, and boundary and initial conditions) have been carried out. All the tests and comparisons similar to those shown in Figures 3 and 4 indicate that the proposed new weighting scheme or the skin-cell simulation is able to closely match analytical solutions for unsaturated radial 
flow problems, while in general the traditional weighting method cannot. It should be mentioned that as shown in Figures 3 and 4, there are certain errors or discrepancies even with the "good" matches between numerical and analytical results. These discrepancies are caused primarily by the coarse spatial discretization of matrix blocks. For example, the double-porosity grid used in the numerical models cannot resolve better approximation of pressure gradients or flow rates near fracture-matrix interfaces, which contributes to the numerical errors shown in Figures 3 and 4. Further discussions of the spatial discretization errors are beyond the scope of this paper.

\subsection{Comparison with Laboratory Experimental Results}

Kazemi (1979) presented a series of laboratory experimental results of water imbibition into fractured matrix cores to displace oil. The laboratory tests were conducted on three sets of artificial fractured cores using cylindrical and rectangular blocks, with one fracture along the long axis for each set. The cylindrical and rectangular matrix blocks were actually cut from Berea sandstone. The laboratory model we consider here consists of a fractured core with two brick-type matrix blocks. Each matrix block has a brick shape with dimension of width, height, and length $(50.8 \times 50.8 \times 101.6 \mathrm{~mm}$ 's $)$ as

shown in Figure 5. The fracture formed between the two matrix cores has an aperture of $0.30 \mathrm{~mm}$. The experimental data used in this study was from Test 38423 (Kazemi, 1979) as an example. In the experiment, flow channels were left open only at the inlet and outlet ends of the fracture (i.e., for water injection and for oil and water flow out), and side fracture and matrix surfaces were sealed. Initially, the fracture and matrix system was fully saturated uniformly with oil (diesel), and then water was injected with a constant rate at the inlet (Figure 5) to displace the oil.

Basic model experimental and modeling parameters are listed in Table 4-3. The relative permeability and capillary pressure curves used are shown in Figures 6 and 7 , respectively. Note that relative permeability curves for both fracture and matrix, shown in Figure 6, were estimated using the equations given in Kazemi (1979), and the matrix capillary pressure curve was taken from the capillary-pressure curve on the Berea core of 
Figure 12 in Kazemi (1979). However, several important parameters were not provided in Kazemi (1979), including residual water saturation, residual oil saturation, and fracture capillary pressure curves. Actual values used for these missing parameters were determined in this work by model calibration, with the final estimates given in Table 4-3 and Figures 6 and 7.

Here, this test is analyzed using a double-porosity approach (equivalent to the explicit-fracture model in this case) to examine the numerical scheme for handling fracture-matrix interaction under multiphase flow conditions. The fracture-matrix set of Figure 5 is treated as a 2-D system along the longitudinal (x) direction (from inlet to outlet). Because of the symmetry, only half of the 2-D model domain (one matrix block and half the fracture) is discretized into a double-porosity grid, using a 1-D parallel fracture concept, with one (actually half) fracture element corresponding to one matrix element in the transverse direction (perpendicular to the fracture plane). Along the $\mathrm{x}$ direction, a uniform linear grid of 10 elements is generated for both the fracture and the matrix block, with a uniform grid spacing of $\Delta x=10.16 \mathrm{~mm}$.

We have performed several model calibration analyses to estimate the missing model parameters. The final simulation results using the proposed physical upstream weighting scheme are compared with the laboratory experimental data in Figure 8. Figure 8 shows excellent agreement between measured and simulated volumetric fractional oil recovery versus pore volume of water injected. This result indicates that the proposed new upstream mobility-weighting scheme is able to capture the main factors that control fracture-matrix interaction during the oil-water displacement for this test problem.

\section{Summary and Concluding Remarks}

We have presented a physically based upstream weighting scheme for modeling multiphase fracture-matrix flow and interaction. This new approach is based on continuity of capillary pressure at the fracture-matrix interface in estimating physically correct relative permeability for multiphase flow between fractures and the matrix. This new conceptual model overcomes a serious flaw that exists in most current simulation 
practice when estimating flow mobility for fracture-matrix flow terms using the conventional upstream weighting method. Numerically, the new scheme uses exactly the same dual-continuum grids, which will otherwise be used with the traditional model approaches, without requiring an additional computational burden or using refined grids, to achieve not only accurate but also physically correct results for fracture-matrix interaction.

To verify the proposed mobility-weighting scheme, we carried out two theoretical studies in this work. The new mobility-weighting method was used to simulate an imbibition process into a single unsaturated matrix cube, and unsaturated flow in a radially fractured formation with fully transient fracture-matrix interaction. In both cases, the proposed physical upstream weighting scheme is found to provide accurate simulation results when compared with analytical solutions. It is also found that the conventional weighting scheme will result in significant errors in estimating fracture-matrix flow in modeling the same physical processes. In the third case, we sought to match published laboratory results for oil-water displacement through a fractured core. Our proposed approach proved able to match laboratory experimental results of fracture-matrix interaction.

An alternative numerical technique to enforce relative permeability consistent with capillary continuity at fracture-matrix interfaces was also explored. It consists of employing an additional gridblock to represent a thin matrix interface layer with the traditional upstream weighting scheme. This paper shows that adding a skin-layer cell to matrix blocks, as long as the volume of the skin cell is sufficiently small or the mobjlity between fractures and the skin cell is large, could achieve the similar results the proposed new weighting scheme.

In this work, we demonstrate that the proposed, physical upstream weighting method is accurate, numerically efficient, and easy to implement into existing dual-or multiple-continuum reservoir simulators. It is recommended as a valid modeling approach for field applications with both dual-continuum and discrete fracture models. 


\section{Acknowledgments}

The authors would like to thank H. H. Liu and Dan Hawkes for their review of the manuscript. This work was supported in part by the Director, Office of Civilian Radioactive Waste Management, U.S. Department of Energy, through Memorandum Purchase Order QA-B004220RB3X between Bechtel SAIC Company, LLC and the Ernest Orlando Lawrence Berkeley National Laboratory (Berkeley Lab). The support is provided to Berkeley Lab through the U.S. Department of Energy Contract No. DEAC03-76SF00098.

\section{Appendix A. Derivation of Analytical Fracture-Matrix Flux}

An analytical solution describing transient unsaturated flow is derived for rigorous treatment of fracture-matrix interaction in a uniform fractured radial formation (Wu and Pan, 2004). The analytical solution relies on the assumption that the specially correlated relative permeability and capillary functions, Equations (4-1) and (4-2), are satisfied, respectively, for fractures and the matrix. In addition, it further requires that the two exponential parameters are specified as $\alpha=2$ and $\beta=1$ in Equations (4-1) and (4-2). Under these conditions, along constant liquid saturation at the wellbore boundary and constant initial saturations in both fracture and matrix systems, the analytical solution is given in the Laplace space (Wu and Pan, 2004) as

$$
\bar{S}_{\mathrm{MD}}=\frac{\mathrm{C}_{\mathrm{pM}}}{\mathrm{C}_{\mathrm{pF}}} \frac{\overline{\mathrm{S}}_{\mathrm{FD}}}{\mathrm{x}_{\mathrm{D}}} \frac{\sinh \left(\sigma \mathrm{x}_{\mathrm{D}}\right)}{\sinh (\sigma)}
$$

and

$$
\bar{S}_{\mathrm{FD}}=\frac{\mathrm{S}_{0 \mathrm{D}}}{\mathrm{p}} \frac{\mathrm{K}_{0}\left(\sqrt{\mathrm{x}_{2} \mathrm{r}_{\mathrm{D}}}\right)}{\mathrm{K}_{0}\left(\sqrt{\mathrm{x}_{2}}\right)}
$$


where $\overline{\mathrm{S}}_{\mathrm{MD}}$ and $\overline{\mathrm{S}}_{\mathrm{FD}}$ are the normalized matrix and fracture saturations, respectively, in the Laplace space, defined similarly by Equation (4-3); $C_{p M}$ and $C_{p F}$ are coefficients $C_{p}$ of the capillary function, Equation (4-2), for matrix and fracture, respectively; $\mathrm{p}$ is the Laplace variable; $S_{0 D}$ is the normalized fracture saturation at the well; $\sigma=\sqrt{A_{3} p}, K_{0}$ is the modified Bessel function of the second kind of zero order; and $\mathrm{x}_{2}$ is defined as:

$$
x_{2}=A_{1} A_{4}[\sigma \operatorname{coth} \sigma-1]+A_{2} p
$$

with

$$
A_{1}=\frac{12 D_{M} \phi_{M} r_{w}^{2}}{D_{f} \phi_{F} B^{2}} \frac{1-S_{M r}}{1-S_{F r}}, A_{2}=\frac{4 r_{w}^{2}}{B^{2}} \text {, and } A_{3}=\frac{D_{F}}{D_{M}}
$$

where subscript $M$ denotes matrix and $F$ for fracture; $r_{w}$ is the well radius; $B$ is fracture spacing or the dimension of matrix cubes $(\mathrm{m}) ; \phi_{\mathrm{M}}$ and $\phi_{\mathrm{F}}$ are matrix and fracture porosities; $\mathrm{S}_{\mathrm{Mr}}$ and $\mathrm{S}_{\mathrm{Fr}}$ are residual saturations, respectively, of fracture and matrix systems; and $\mathrm{D}_{\mathrm{F}}$ or $D_{M}$ is the moisture diffusivity term for fracture or matrix, respectively, defined (Wu and Pan, 2004) as:

$$
D_{\xi}=\frac{k_{\xi} k_{r \xi}}{\phi_{\xi} \mu_{w}} \frac{\partial P_{w \xi}}{\partial S_{w}}=\frac{k_{\xi} C_{k \xi} C_{p \xi}}{\phi_{\xi} \mu_{w}\left(1-S_{\xi r}\right)}
$$

with a dimension of $\mathrm{m}^{2} / \mathrm{s}$. Subscript $\xi$ is an index for fracture $(\xi=F)$ or matrix $(\xi=M) ; C_{k} \xi$ and $C_{k} \xi$ are coefficients $C_{k}$ of the relative permeability function, Equation (4-1), for matrix and fracture, respectively.

The analytical solution uses the following dimensionless variables: dimensionless radial distance, dimensionless spherical distance inside matrix blocks, and dimensionless time: 
$r_{D}=\frac{r}{r_{w}}, \quad x_{D}=\frac{2 x}{B}, \quad$ and $\quad t_{D}=\frac{D_{F} t}{(B / 2)^{2}}$

where $\mathrm{r}$ is the radial distance from the well; $\mathrm{x}$ is the distance from a nested cross sectional surface within matrix blocks (having an equal distance to the matrix surface or equivalent to spherical radial distance) to the center of cube; and $t$ is time.

From the analytical solutions, Equations (A-1) and (A-2), the fracture-matrix mass flux in the Laplace space can be derived from its definition:

$$
\overline{\mathrm{q}}_{\mathrm{FM}}=-\left.\frac{\mathrm{k}_{\mathrm{M}} \mathrm{k}_{\mathrm{rM}}}{\mu_{\mathrm{w}}} \rho_{\mathrm{w}} \frac{\partial \mathrm{P}_{\mathrm{w}}}{\partial \mathrm{x}}\right|_{\mathrm{x}=\mathrm{B} / 2}=\frac{\mathrm{k}_{\mathrm{M}} \rho_{\mathrm{w}} \mathrm{C}_{\mathrm{pM}}^{2} \mathrm{C}_{\mathrm{kM}}}{\mu_{\mathrm{w}} \mathrm{B} \mathrm{C}_{\mathrm{pF}}} \overline{\mathrm{S}}_{\mathrm{FD}}[\sigma \operatorname{coth} \sigma-1]
$$

where $\overline{\mathrm{q}}_{\mathrm{FM}}$ is the Laplace transformed transient fracture-matrix flux and a unit of $\mathrm{kg} / \mathrm{s} / \mathrm{m}^{2}$ in real space as a function of time and radial distance. Similarly, a cumulative fracture-matrix mass flow or exchange rate $\left(\mathrm{Q}_{\mathrm{FM}}\right)$ can be derived as

$$
\mathrm{Q}_{\mathrm{FM}}=\int_{0}^{\mathrm{t}} \mathrm{q}_{\mathrm{FM}}(\mathrm{r}, \mathrm{t}) \mathrm{dt}
$$

In the Laplace space, the cumulative rate is determined by

$$
\overline{\mathrm{Q}}_{\mathrm{FM}}=\frac{(\mathrm{B} / 2)^{2}}{\mathrm{D}_{\mathrm{F}}} \frac{\overline{\mathrm{q}}_{\mathrm{FM}}}{\mathrm{p}}
$$

Note the cumulative fracture-matrix mass flow rate has a unit of $\mathrm{kg} / \mathrm{m}^{2}$, which is not the net amount of mass exchange, but an integrated exchange rate per unit area.

\section{References}

Aziz, K. and Settari A., 1979. Petroleum Reservoir Simulation, Applied Science Publishers LTD, London. 
Barenblatt, G.I., Zheltov I.P., and Kochina I.N., 1960. Basic concepts in the theory of seepage of homogeneous liquids in fissured rocks, PMM, Sov. Appl. Math. Mech., 24(5), 852-864.

Berkowitz, B., 2002. Characterizing flow and transport in fractured geological media: A Review, Advance in Water Resources,25, 861-884.

Brooks, R.H. and Corey A.T., 1964. Hydraulic properties of porous media, Hydrology Papers, No. 3, Colorado State University, Ft. Collins, Colo.

Forsyth, P.A. andKropinski M.C. 1997. Monotonicisy considerations for saturatedunsaturated subsurface flow, SIAM. J. Sci. Comput., 18(5), 1328-1354.

Forsyth, P.A., Wu, Y.S., and Pruess, K. 1995. Robust numerical methods for saturatedunsaturated flow with dry initial conditions in heterogeneous media, Advance in Water Resources, 18, 25-38.

Huyakorn, P. S. and Pinder G. F., 1983, Computational Methods in Subsurface Flow, Academic Press, Inc., New York.

Kazemi, H., 1979. Numerical simulation of water imbibition in fractured cores, Soc. Pet. Eng. J. 323-330.

Kazemi, H., 1969. Pressure transient analysis of naturally fractured reservoirs with uniform fracture distribution. Soc. Pet. Eng. J., 451-62. Trans., AIME, 246.

Lai, C.H., Bodvarsson G.S., Tsang C.F., and Witherspoon P.A., 1983. A new model for well test data analysis for naturally fractured reservoirs, SPE-11688, Presented at the 1983 California Regional Meeting, Ventura, California, March.

Peaceman, D. W., 1977, Fundamentals of Numerical Reservoir Simulation, Developments in

Petroleum Sciences, 6, Elsevier Scientific Publishing Company, Amsterdam-Oxford-New York.

Persoff, P. and Pruess K., 1995. Two-phase flow visualization and relative permeability measurements in natural rough-walled rock fractures, Water Resources Research, $31(5), 1175-1186$. 
Pruess K., Oldenburg, C., and Moridis, G., 1999. TOUGH2 User's Guide, Version 2.0, Report LBNL-43134, Berkeley, California: Lawrence Berkeley National Laboratory.

Pruess, K., and Narasimhan, T.N., 1985. A practical method for modeling fluid and heat flow in fractured porous media, Soc. Pet. Eng. J., 25, 14-26.

Pruess, K., 1983. GMINC - A mesh generator for flow simulations in fractured reservoirs, Report LBL-15227, Berkeley, California: Lawrence Berkeley National Laboratory.

Richards, L. A., 1931. Capillary conduction of liquids though porous medium, Physics, 1, 318-333.

Snow, D.T., 1965. A parallel plate model of fractured permeable media, Ph.D. Dissertation, 331. pp., University of California, Berkeley.

Stothoff, S. and Or D., 2000.A discrete-fracture boundary integral approach to simulating coupled energy and moisture transport in a fractured porous medium, "Dynamics of Fluids in Fractured Rocks, Concepts and Recent Advances", Edited by B. Faybishenko, P. A. Witherspoon and S. M. Benson, AGU Geophysical Monograph 122, American Geophysical Union, Washington, DC, 269-279.

van Genuchten, M. Th., 1980. A closed-form equation for predicting the hydraulic conductivity of unsaturated soils, Soil Sci. Soc. Amer. J, 44(5), 892-898.

Warren, J.E., and Root P.J., 1963. The behavior of naturally fractured reservoirs, Soc. Pet. Eng. J., pp. 245-255, Trans., AIME, 228.

$\mathrm{Wu}$, Y.S. and Pan L., 2004. Analytical solutions for transient flow through unsaturated fractured porous media, Proceedings of the Second International Symposium on Dynamics of Fluids in Fractured Rocks, Edited by B. Faybishenko and P. A. Witherspoon, Lawrence Berkeley National laboratory, University of Californian, Berkeley, Californian, USA, 360-366, February 10-12.

$\mathrm{Wu}$, Y.S. and Pan L., 2003. Special relative permeability functions with analytical solutions for transient flow into unsaturated rock matrix, Water Resources Research, 39 (4) 3-1-3-9. 
Wu, Y.S., 2000a. On the effective continuum method for modeling multiphase flow, multicomponent transport and heat transfer in fractured rock, "Dynamics of Fluids in Fractured Rocks, Concepts and Recent Advances", Edited by B. Faybishenko, P. A. Witherspoon and S. M. Benson, AGU Geophysical Monograph 122, American Geophysical Union, Washington, DC, 299-312.

Wu, Y. S., 2000b. A virtual node method for handling wellbore boundary conditions in modeling multiphase flow in porous and fractured media, Water Resources Research, 36 (3), 807-814.

Wu, Y.S. and Pruess K., 2000. Numerical simulation of non-isothermal multiphase tracer transport in heterogeneous fractured porous media, Advance in Water Resources, $23,699-723$.

Wu, Y. S, Haukwa C., and Bodvarsson G. S., 1999. A Site-Scale Model for Fluid and Heat Flow in the Unsaturated Zone of Yucca Mountain, Nevada. Journal of Contaminant Hydrology. 38 (1-3), 185-217.

Wu, Y.S., Ahlers C.F., Fraser P., Simmons A., and Pruess K., 1996. Software qualification of selected TOUGH2 modules, Report LBNL-39490, Lawrence Berkeley National Laboratory, Berkeley, CA.

Wu, Y.S. and Pruess K., 1988. A multiple-porosity method for simulation of naturally fractured petroleum reservoirs, SPE Reservoir Engineering, 3, 327-336. 